\title{
Multiple System Atrophy
}

National Cancer Institute

\section{Source}

National Cancer Institute. Multiple System Atrophy. NCI Thesaurus. Code C84909.

A rare neurodeg enerative disorder characterized by loss of autonomic nervous system

functions and disturbances of motor, balance and muscle coordination. 